\title{
A selfish element
}

\section{Genes in Conflict: The Biology of Selfish Genetic Elements \\ by Austin Burt \& Robert Trivers \\ Belknap Press: 2006.610 pp. \$35, £21.95}

\section{James F. Crow}

In the early 1970s, Robert Trivers published six articles on the evolution of social behaviour. They were ignored by most social scientists, who were reluctant to consider natural selection as a cause of human behavioural traits, and they were bitterly attacked by marxists for reasons of doctrine. Some population geneticists also objected, because Trivers did not formulate his concepts as standard equations for changes in allele frequency. Yet the papers have proved seminal, and Trivers is now recognized, along with Edward O. Wilson and Bill Hamilton, as one of the founders of behavioural evolution and sociobiology. A collection of his papers has been published (Natural Selection and Social Theory; Oxford University Press, 2002), along with personal histories that add greatly to their interest. Young scientists will be empathetic: both Hamilton and Trivers had trouble with publishers, grants and critics.

Just over ten years ago, Trivers joined forces with geneticist Austin Burt for a detailed study of selfish genetic elements, and Genes in Conflict is the result of their fruitful collaboration. The book is the first of its kind and admirably fills an empty niche.

The mechanism of meiosis strongly selects against elements that violate Mendel's rules, yet there are clever tricksters that manage to beat the system. Some of these are well known, but it is surprising how many there are. There seems to be no molecular or cellular process that cannot be subverted by a sufficiently clever opportunist. The book starts with the wellstudied $t$-alleles in the mouse and segregation distortion in fruitflies. Successive chapters then treat selfish sex chromosomes, imprinting, selfish mitochondria, gene conversion, 'homing', transposable elements, B chromosomes, and the exclusion of whole genomes. The book includes some 1,500 references, and several of the scientists who have contributed most to evolutionary explanations are mentioned, of whom David Haig stands out for his inventiveness.

Each of these subjects is treated fully, with their detailed cellular mechanisms described and, when they are known, the molecular explanations. An important feature of the book is that each subject is treated from an evolutionary viewpoint, as you might expect from these authors. Their views are imaginative but are sometimes speculative or difficult to test; they are great if you don't inhale. Yet for me, this is what makes the book especially interesting - it is far more stimulating than a series of descriptions. Tables that summarize the facts will help readers keep the details straight, and the many diagrams will aid their understanding of the mechanisms. Even so, I needed to have a pencil and paper handy. The authors also raise practical possibilities of the work, such as engineering driven Y chromosomes to control insect numbers.

The authors emphasize that the selfish entities they describe are almost always harmful to the host. They have little patience with those who assume an adaptive significance, including even Barbara McClintock, dismissing her idea that transposons - segments of DNA that can move around the genome - act as 'controlling elements'. Despite this, some elements have been domesticated and do, in fact, serve a useful purpose. Some of the most interesting speculation surrounds the possible origin of normal processes from selfish elements in the past. Two of the most convincing are mating-type switching in yeast and telomeres in several species.

The chapter on selfish cell lineages focuses on cancers as the most obvious example. The authors report the work of Andrew Wilkie and colleagues, who explained the high mutation rate in Apert's syndrome (a condition involving the premature fusion of bones). It is caused by a switch in the male germ line from asymmetric stem-cell divisions, in which the daughter cells are different, to symmetric exponential growth, which greatly increases the number of mutant cells. This highlights the susceptibility of a cell line in which one daughter product undergoes apoptosis to subversion by exponentially growing variants. There must be an evolutionary trade-off between the advantages of asymmetric growth (providing a constant number of sperm continuously over a long lifetime) and a consequent high rate of deleterious mutation. Asymmetric cell division seems to be a prime target for subversion by exponentially growing derivatives. There are likely to be more examples waiting to be found, especially ones that do little harm.

The book ends with a thoughtful chapter on the present position and on future directions of study. Although many of the selfish elements so fully described in the book have lost their motility and died (sometimes increasing the size of the genome), the subject is very much alive.

James F. Crow is professor emeritus of genetics at the University of Wisconsin-Madison, 425G Henry Mall, Madison, Wisconsin 53706, USA.

\section{Inside knowledge}

The science of anatomy reached a peak in nineteenth-century Paris, where anatomist Jean Baptiste Marc

Bourgery and illustrator Nicolas Henri Jacob completed a monumental opus on anatomy and surgery.

The eight-volume work, which incorporates more than 700 illustrations of remarkable clarity and distinctive style, took two decades to complete.

The plates are now reproduced in their entirety in the Atlas of Human Anatomy and Surgery (Taschen, $€ 100, \$ 200$, €150), which has accompanying text in French, English and German. A.A.

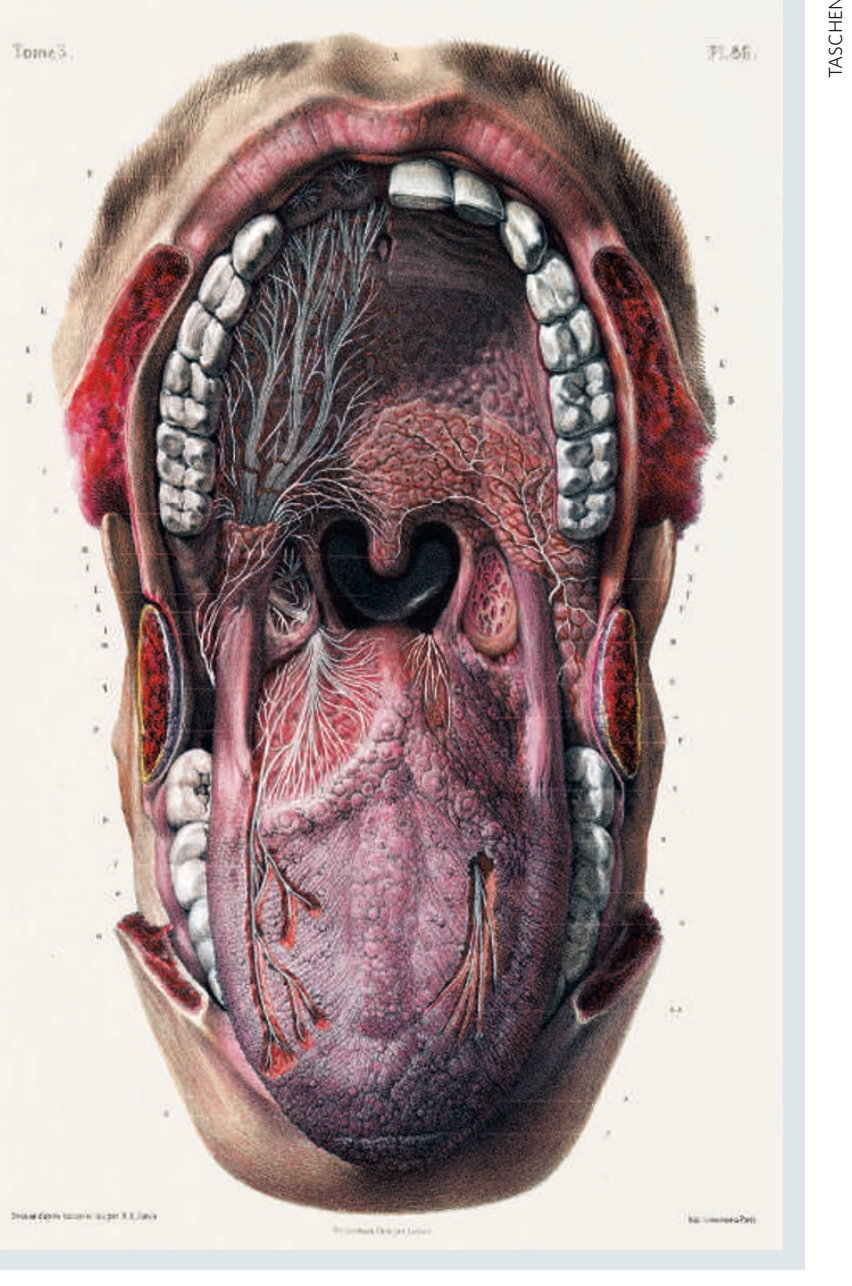

